\title{
Morphology, Optical and AC Electrical Properties of Copper Phthalocyanine Thin Films
}

\author{
M.E. Azim-Araghi, S. Haji Mirza Mohamadi and Z. Bisadi* \\ Applied Physics Division, Physics Department, Kharazmi University, 43 Mofateh Av., Tehran, Iran
}

(Received April 16, 2013; in final form September 30, 2013)

\begin{abstract}
AC electrical properties of sandwich devices composed of thermally evaporated thin films of copper phthalocyanine $(\mathrm{CuPc})$ with aluminum and gold electrodes $(\mathrm{Al} / \mathrm{CuPc} / \mathrm{Au})$ are investigated over frequency $(f)$ range of $10^{2}-10^{5} \mathrm{~Hz}$ and temperature range of $293-453 \mathrm{~K}$. Morphology of the samples was studied via field emission scanning electron microscope images and X-ray diffraction micrographs. The X-ray diffraction micrograph indicates the configuration of $\alpha-\mathrm{CuPc}$ with the (510) plane as the preferred orientation. UV-Vis absorption spectrum was analyzed and the optical band-gap energy of CuPc thin film was determined to be $2.81 \pm 0.01 \mathrm{eV}$. Capacitance increased with increasing temperature especially for $f=10^{2} \mathrm{~Hz}$. Loss factor decreased considerably with increasing frequency to a minimum value at about $f=10^{4} \mathrm{~Hz}$ and increased afterwards. Capacitance is generally independent of frequency for $T \leq 413 \mathrm{~K}$; however it decreases remarkably with increasing frequency for $T>413 \mathrm{~K}$. The conductivity increases quite noticeably with increasing frequency particularly for $T \leq 413 \mathrm{~K}$. The AC electrical characteristics are in good agreement with Goswami and Goswami model. According to our data, at high temperatures, the band theory is applicable in describing the conduction process, whereas hopping mechanism is dominant at low temperatures.
\end{abstract}

DOI: 10.12693/APhysPolA.125.87

PACS 73.40.Sx, 73.90.+f, 72.20.Ee, 72.80.Le

\section{Introduction}

Metal phthalocyanines (MPcs) are organic semiconducting materials which have been widely employed as active layers in gas sensing applications over the last two decades [1-3]. They have been also used in photovoltaic devices [4] and nonlinear optics [5]. Organic semiconductor thin films exhibit a high potential for production of efficient, low cost, and flexible electronic and optoelectronic devices with the option for large area applications [6]. In this type of materials, the $\pi$-orbitals overlap of adjacent organic molecules contributes to the electrical transport [7].

Halogenated Pcs, composites of conductive polymers and Pcs, and heterojunctions of Pcs have been studied for their gas sensing properties on exposure to different gases such as $\mathrm{NO}_{2}, \mathrm{CO}_{2}$, and $\mathrm{O}_{2}$ [8-10].

Copper phthalocyanine $(\mathrm{CuPc})$ is a type of $\mathrm{MPc}$ whose structural [11], electrical [12] and gas sensing [2, 13-15] properties have been under intense investigation. Hassan and Gould [16] studied the effect of oxygen exposure and annealing on the conductivity of $\mathrm{CuPc}$ thin films and reported a discrete trap level at higher voltages, which was attributed to oxygen incorporation within the films.

In this study we intend to investigate the $\mathrm{AC}$ electrical characteristics of $\mathrm{CuPc}$ sandwich devices with $\mathrm{Al}$ and $\mathrm{Au}$ electrodes before and after exposure to oxygen on which little work has been reported so far. We study the annealing effect on the electrical properties of the de-

*corresponding author; e-mail: bisadi.zahra@gmail.com vices, as well. The optical properties and morphology of samples are also analyzed using UV-Vis absorption spectroscopy, scanning electron microscopy (SEM), and $\mathrm{X}$-ray diffraction (XRD) analyses.

\section{Experimental}

Sandwich devices were fabricated using thermal evaporation method under a vacuum of approximately $10^{-5}$ mbar with the evaporation rate of $0.8 \mathrm{~nm}$ per second. First, $70 \mathrm{~nm}$ of gold was evaporated on a glass substrate to act as the lower electrode. $1 \mu \mathrm{m}$ of $\mathrm{CuPc}$ was then evaporated on the gold layer. Finally $70 \mathrm{~nm}$ of aluminum was evaporated onto the $\mathrm{CuPc}$ layer to act as the upper electrode. The sandwich devices produced in this way had an area of $10 \times 10 \mathrm{~mm}^{2}$.

The $\mathrm{AC}$ electrical measurements were then taken in the dark condition via MT4080A Motech LCR multi-frequency meter over the temperature range of $T=293$ to $453 \mathrm{~K}$ and the frequency range of $10^{2}-10^{5} \mathrm{~Hz}$ before and after exposure to oxygen with pressure of 0.5 mbar. We investigated the morphology and crystalline structure of $\mathrm{CuPc}$ thin films by field emission scanning electron microscope (FESEM) images and XRD patterns using Hitachi, Japan S-4160 and INEL, France Equinox 3000 equipment, respectively. We also studied the optical properties of $\mathrm{CuPc}$ thin films via Perkin Elmer, Lambda $25 \mathrm{UV} /$ Vis Spectrometer.

\section{Results and discussion}

\subsection{Structural characteristics}

FESEM images of CuPc thin films with different magnifications are shown in Fig. 1a and b. The estimated 
average size of the particles is $90-100 \mathrm{~nm}$ and a quite uniform distribution of the roughly spherical-shaped particles is observed in Fig. 1a and b.

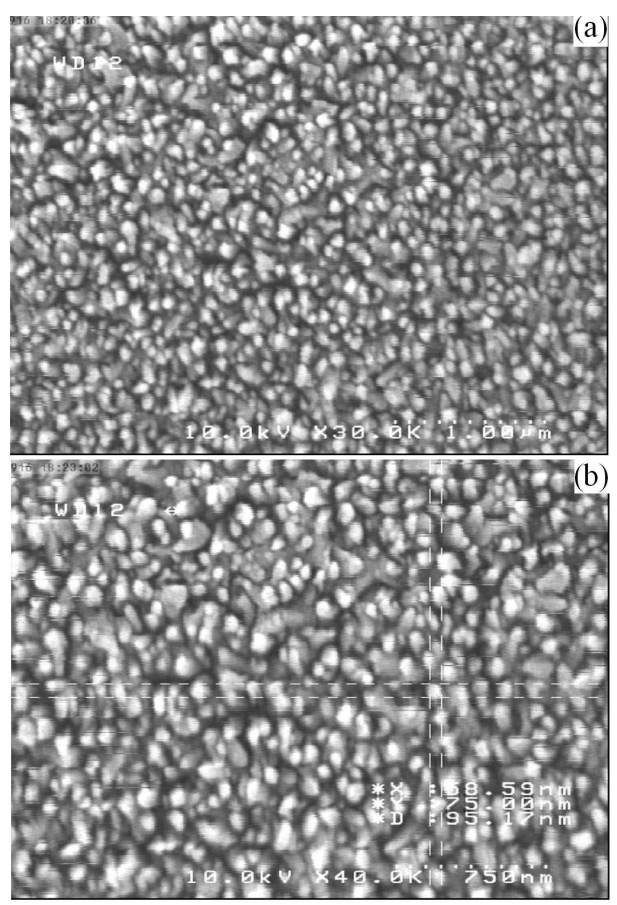

Fig. 1. FESEM images of the surface of $\mathrm{CuPc}$ thin film at (a) $1 \mu \mathrm{m}$ and (b) $750 \mathrm{~nm}$ magnifications indicating the mean particle diameter to be $90-100 \mathrm{~nm}$.

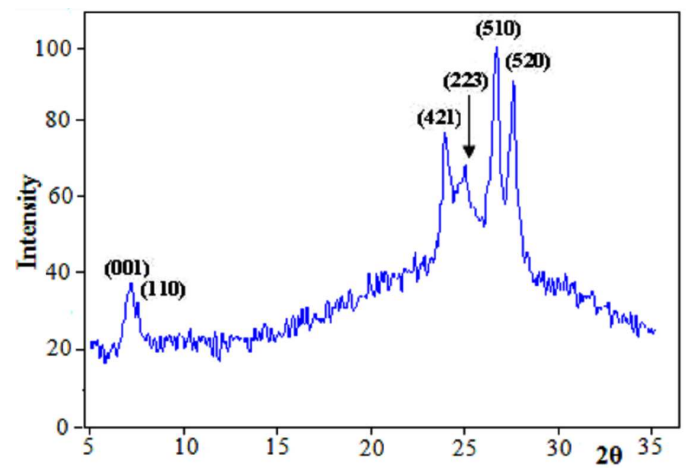

Fig. 2. X-ray diffraction micrograph of $\alpha-\mathrm{CuPc}$ thin film with the thickness of $1 \mu \mathrm{m}$.

The X-ray diffraction pattern of the $\mathrm{CuPc}$ thin films is illustrated in Fig. 2. The XRD micrograph in Fig. 2 indicates the configuration of $\alpha$-form with the (510) plane as the preferred orientation. The numerical Ref. [17] and measured data are presented in Table I. The reference and measured intensity values are noticed to be different particularly for the first two peaks. Discrepancies in the relative peak intensities between conventional diffraction and X-ray diffraction patterns are largely due to the texture effect [18]. The mean crystalline size was calculated
TABLE I

X-ray diffraction data for $\alpha$-CuPc sample at $25 \pm 1{ }^{\circ} \mathrm{C}^{a}$.

\begin{tabular}{|c|c|c|c|c|}
\hline$h k l$ & $2 \theta_{\mathrm{r}}$ & $2 \theta_{\mathrm{m}}$ & $I_{\mathrm{r}}$ & $I_{\mathrm{m}}$ \\
\hline $\begin{array}{llll}0 & 0 & 1\end{array}$ & 6.906 & 7.005 & 100 & 39.58 \\
\hline 110 & 7.246 & 7.353 & 90 & 33.75 \\
\hline 421 & 24.184 & 23.938 & 70 & 72.78 \\
\hline 223 & 25.522 & 25.025 & 70 & 64.73 \\
\hline 510 & 26.210 & 26.619 & 90 & 100 \\
\hline 520 & 27.616 & 27.510 & 90 & 90.94 \\
\hline
\end{tabular}

${ }^{a}$ Miller indices and diffraction angles (Ref. [17] (r) and measured $(\mathrm{m})$ for $\alpha-\mathrm{CuPc})$ are given.

The intensities $I_{\mathrm{r}}$ and $I_{\mathrm{m}}$ refer to the peak height.

by Scherrer's formula [19]:

$$
L=\frac{k_{\mathrm{S}} \lambda}{\beta_{0} \cos \theta},
$$

where $\lambda$ is the $\mathrm{X}$-ray wavelength $(1.540 \AA), \beta_{0}$ - the full width at half maximum (FWHM) of the most intense peak in radians, $\theta-$ Bragg's angle and $k_{\mathrm{S}}-$ the Scherrer's constant $=0.9$ [19]. The mean crystalline size was calculated to be about $82 \mathrm{~nm}$ which is in quite good agreement with the estimated value obtained from FESEM images.

\subsection{Optical properties}

The optical absorption spectrum of $\mathrm{CuPc}$ thin films is illustrated in Fig. 3. The UV-Vis spectrum for phthalocyanines originates from molecular orbitals within the aromatic $18 \pi$ electron system and from overlapping orbitals on the central metal [20]. The conjugated double bonds within the crystal structure of the film create electron orbitals which overlap between molecular $\pi$ orbitals. These electrons are able to transfer energy throughout the structure and are responsible for the absorption peaks [21].

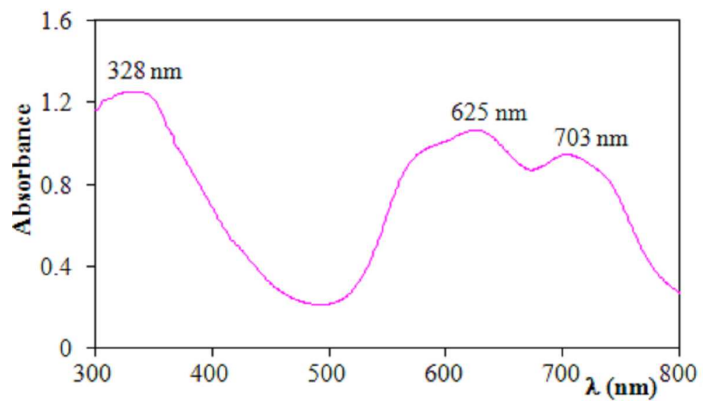

Fig. 3. UV-Vis absorption spectrum of $\mathrm{CuPc}$ thin film with the thickness of $1 \mu \mathrm{m}$.

A doublet of peaks exists in the visible region in Fig. 3 at 625 and $703 \mathrm{~nm}$ known as the $Q$-bands. In the near UV region a single peak is observed at $328 \mathrm{~nm}$ ( $B$-band) and it seems that there are two trap levels with 1.76 and $1.99 \mathrm{eV}$. The absorption coefficient $\alpha$ can be calculated using the relation below 


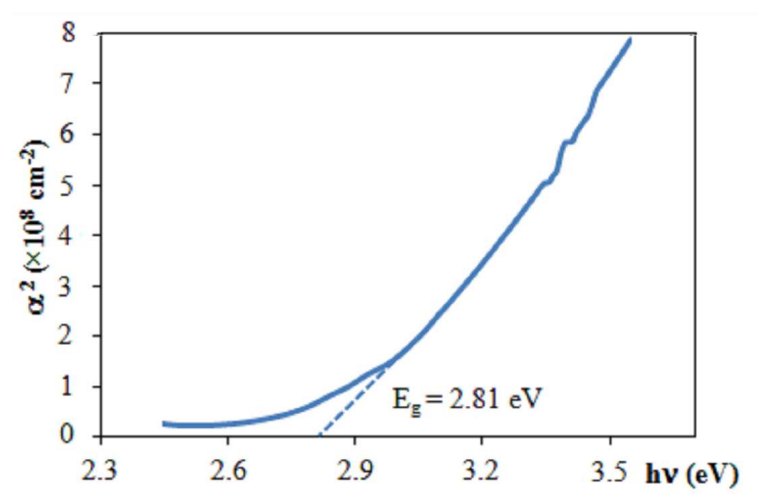

Fig. 4. $\quad \alpha^{2}$ as a function of photon energy $(h \nu)$ indicating a band gap of $2.81 \pm 0.01 \mathrm{eV}$ for CuPc thin films.

$$
\alpha=2.303 \frac{A}{t},
$$

where $A$ is the absorbance of the film and $t$ is its thickness. For direct allowed transition, the absorption coefficient $\alpha$ is related to the photon energy $(h \nu)$ by the following relation:

$$
\alpha=\alpha_{0}\left(h \nu-E_{\mathrm{g}}\right)^{1 / 2},
$$

where $E_{\mathrm{g}}$ is the optical bandgap and $\alpha_{0}$ is a constant [22]. In Fig. $4, \alpha^{2}$ is plotted as a function of photon energy $(h \nu)$ which indicates the existence of a direct optical bandgap of $2.81 \pm 0.01 \mathrm{eV}$ for $\mathrm{CuPc}$ thin films.

\section{3. $A C$ measurements}

3.3.1. Frequency and temperature effects on conductivity

Figure 5 shows the dependence of conductivity $(\sigma)$ on frequency $(f)$ at different temperatures $(T)$. The conductivity is observed to be strongly dependent on frequency especially for $T \leq 413 \mathrm{~K}$. This dependence of conductivity on frequency can be explained by the predominance of the hopping mechanism [23]. Hopping electrons possess a wide range of relaxation times and as a result the conductivity should increase with increasing frequency.

For higher temperatures $T>413 \mathrm{~K}$, conductivity is almost constant for a wide frequency range up to a threshold frequency at which it starts increasing quite considerably. At higher temperatures and low frequency region, the conduction mechanism is mainly of the band type [24].

\section{TABLE II}

Activation energy values for $\mathrm{Au} / \mathrm{CuPc} / \mathrm{Al}$ devices at different frequencies.

\begin{tabular}{c|c|c|c}
\hline \hline \multirow{2}{*}{$\begin{array}{c}\text { Frequency } \\
{[\mathrm{Hz}]}\end{array}$} & \multicolumn{3}{|c}{ Activation energy [eV] } \\
\cline { 2 - 4 } & $E_{1}$ & $E_{2}$ & $E_{3}$ \\
\hline $10^{2}$ & 0.89 & 0.43 & 0.14 \\
$10^{3}$ & 0.85 & 0.36 & 0.12 \\
$10^{4}$ & 0.62 & 0.24 & 0.1 \\
$10^{5}$ & 0.04 & 0.16 & 0.05
\end{tabular}

The conductivity versus $1000 / T$ is illustrated in Fig. 6 for different frequencies. Activation energy is calculated

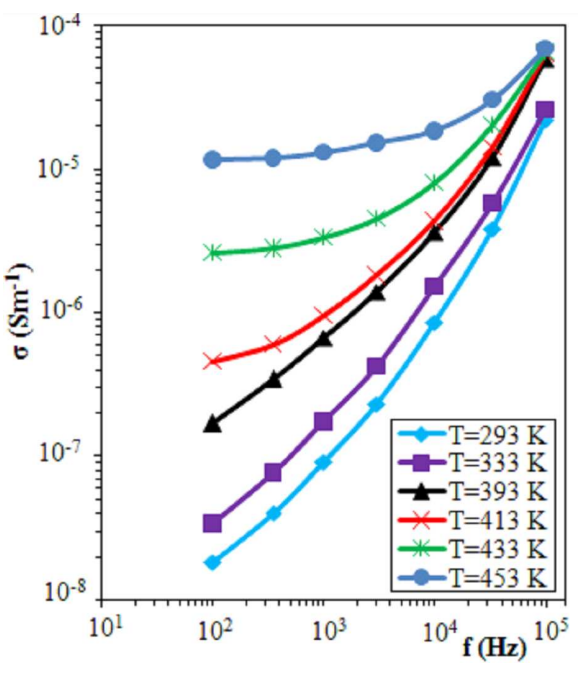

Fig. 5. Variation of AC conductivity with frequency at different temperatures.

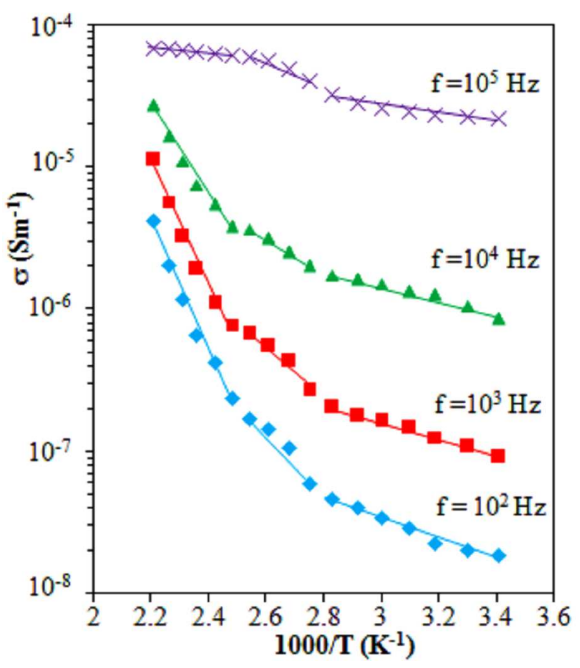

Fig. 6. Variation of AC conductivity with temperature at different frequencies.

from the slope of the plots for three different temperature regions and the obtained results are enumerated in Table II. $E_{1}$ is the activation energy in the higher temperature region and $E_{2}$ and $E_{3}$ are those in the lower temperature region. $E_{1}$ arises from the intrinsic charge carriers when the conduction mechanism is free band type, while $E_{2}$ and $E_{3}$ depend on the extrinsic conduction due to impurity scattering. In the extrinsic conduction region the charge carriers move by hopping along with the ions or electrons [25].

\subsubsection{Oxygen exposure and annealing effects on conductivity}

Figure 7 displays a logarithmic plot of conductivity versus frequency for samples exposed to oxygen gas with the pressure of 0.5 mbar for various exposure times of $24,48,168$, and $336 \mathrm{~h}$ at room temperature. It is ev- 
ident that in the low frequency range, conductivity is strongly dependent on oxygen exposure time. However, it is observed that for frequencies higher than $10^{4} \mathrm{~Hz}$ this dependence no longer exists and all samples exhibit quite the same behavior.

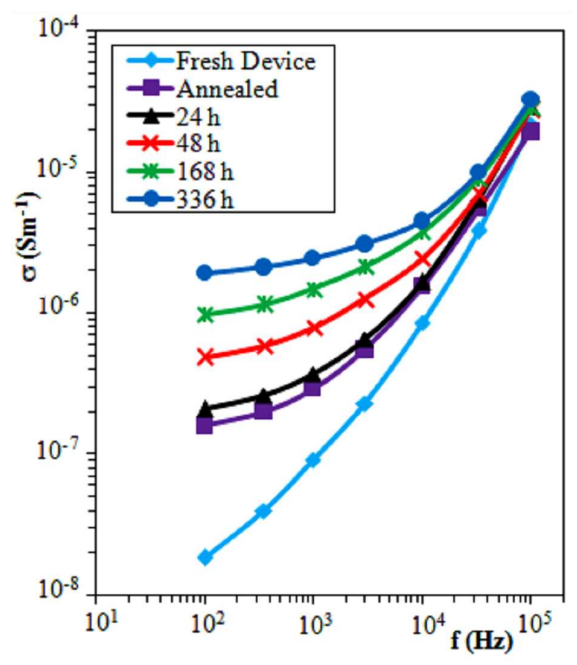

Fig. 7. Variation of AC conductivity with frequency at different exposure times to $\mathrm{O}_{2}$ and after annealing.

The mechanism of oxidation and reduction is an important factor in the increase or decrease in conductivity. The coordination of $\mathrm{O}_{2}$ to $\mathrm{MPc}$ at the air-Pc interface results in the formation of $\mathrm{MPc}^{+}$and $\mathrm{O}_{2}^{-}$and causes the injection of hole charge carriers into the solid [26] and hole concentration near the surface increases. Thus a rise in electrical conductivity is achieved through a doping mechanism [27] at low frequency region. Most Pcs are $p$-type semiconductors and their conductivity is enhanced after exposure to acceptor gases like $\mathrm{O}_{2}$ [10].

The samples which were previously exposed to oxygen for $336 \mathrm{~h}$ went through heat treatment under high vacuum at $T=452 \mathrm{~K}$ for $1 \mathrm{~h}$ and then were cooled down to the room temperature prior to AC electrical characterization. The conductivity versus frequency for fresh devices at room temperature and the annealed samples is also plotted in Fig. 7. It is observed that conductivity of the sample decreases significantly by annealing, but it is still higher than the value obtained for the fresh devices since the complete desorption of $\mathrm{O}_{2}$ molecules from the $\mathrm{CuPc}$ film was not achieved. It is probable that if the annealing temperature was higher and/or the annealing process duration was longer, complete removal of oxygen would occur and the same curve as the fresh device would appear for annealed samples.

3.3.3. Frequency and temperature effects on capacitance

The frequency dependence of capacitance in the temperature range of $293-453 \mathrm{~K}$ is shown in Fig. 8. At relatively high temperatures $(T>413 \mathrm{~K})$ and low frequencies $\left(f<10^{4} \mathrm{~Hz}\right)$, capacitance seems to be strongly dependent on frequency, but at lower temperature and higher frequency range the curves are practically parallel to the frequency axis.

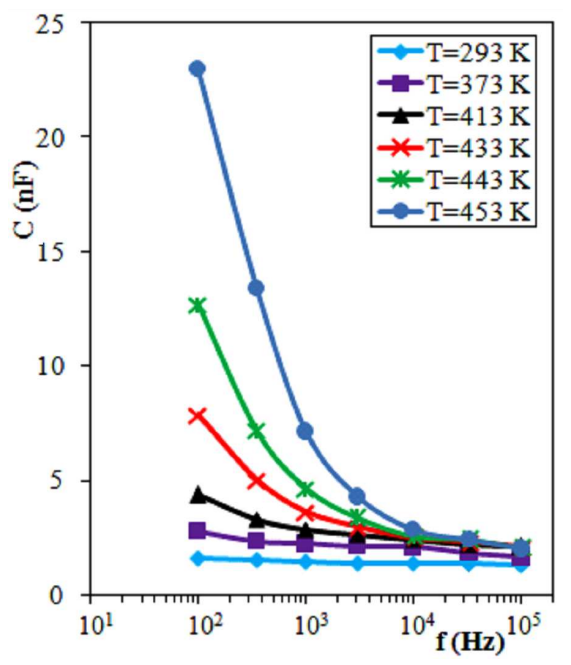

Fig. 8. Variation of capacitance with frequency at different temperatures.

According to Goswami and Goswami model [28] if our capacitor system is assumed to comprise (i) an inherent capacity element $(C)$ unaffected by frequency $(f)$ and temperature, (ii) a discrete resistance element $(R)$ due to the dielectric film in parallel with $C$ and (iii) a series resistance $(r)$ due to lead length, etc., and if the equivalent series circuit $\left(C_{\mathrm{S}}\right.$ and $\left.R_{\mathrm{S}}\right)$ is taken into account, the equivalent series capacitance (with $\omega=2 \pi f$ ) can be written as

$$
C_{\mathrm{s}}=\frac{1+\omega^{2} R^{2} C^{2}}{\omega^{2} R^{2} C}=\frac{1}{\omega^{2} R^{2} C}+C .
$$

If, however, $1 / \omega^{2} R^{2} C \ll C$, then $C_{\mathrm{s}}=C$. This condition can be satisfied in a capacitor system either by increasing $R$ (i.e. by lowering of the temperature) or by raising $\omega$. A suitable combination of these factors will lead to the frequency independent capacitance [28].

The capacitance as a function of temperature is plotted in Fig. 9. It can be clearly seen that at high temperatures and for frequencies less than $10^{4} \mathrm{~Hz}$, capacitance increases considerably with increasing temperature particularly for $f=10^{2} \mathrm{~Hz}$.

\subsubsection{Oxygen exposure and annealing effects on capacitance}

The variation of capacitance with frequency for samples exposed to oxygen for various exposure times is plotted in Fig. 10. It is seen that in low frequency region capacitance changes remarkably with exposure time. The increase of $C_{\mathrm{s}}$ at low frequencies can be ascribed to the increase of the conductivity of $\mathrm{CuPc}$ layer induced by oxygen adsorption which leads to a fall in the value of $R$ in Eq. (4) and thus causes the measured capacitance to rise.

For frequency values higher than $10^{4} \mathrm{~Hz}$, however, all samples show quite the same behavior and capacitance is almost independent of frequency and reaches a minimum 


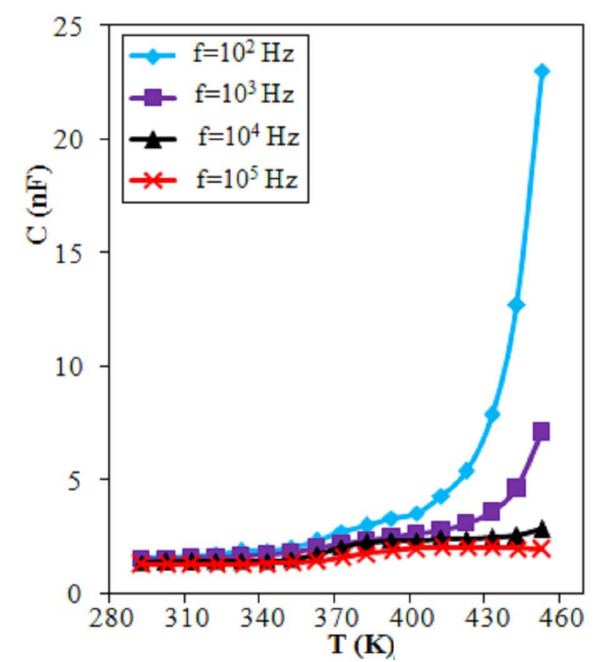

Fig. 9. Variation of capacitance with temperature at different frequencies.

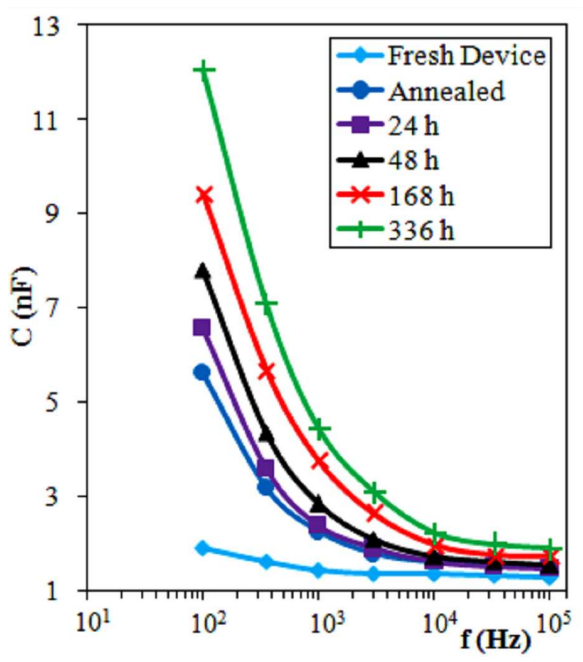

Fig. 10. Variation of capacitance with frequency at different exposure times to $\mathrm{O}_{2}$ and after annealing.

value which is nearly constant for all samples. This effect has been studied in the previous section.

Annealing (at $T=452 \mathrm{~K}$ for $1 \mathrm{~h}$ ) of the samples which were already exposed to $\mathrm{O}_{2}$ for $336 \mathrm{~h}$ has greatly reduced the capacitance of the samples. This effect can be attributed to desorption of oxygen molecules from the $\mathrm{CuPc}$ layer. It is clearly seen in Fig. 10 that annealing has not completely reversed the effect of oxygen adsorption. The difficult process of desorption of oxygen molecules from $\mathrm{CuPc}$ thin film is possibly responsible for this effect. We expect full reversibility for a longer annealing duration.

3.3.5. Frequency and temperature effects on loss factor

The loss factor $(\tan \delta)$ as a function of frequency and temperature is plotted in Figs. 11 and 12. As shown in Fig. 11, loss factor is strongly dependent on frequency particularly at relatively high temperatures and low frequencies. It decreases sharply with increasing frequency to a minimum value at about $f=10^{4} \mathrm{~Hz}$ and increases gradually afterwards. The behavior observed here is in good agreement with Goswami and Goswami model. Referring to this model again, loss factor is proposed as [28]:

$$
\tan \delta=\frac{1}{\omega R C}+\frac{r}{\omega R^{2} C}+\omega r C .
$$

When $\omega R^{2} C \gg r$ or $r / R \ll 1$ which is true for all cases, Eq. (5) is written as

$$
\tan \delta=\frac{1}{\omega R C}+\omega r C \text {. }
$$

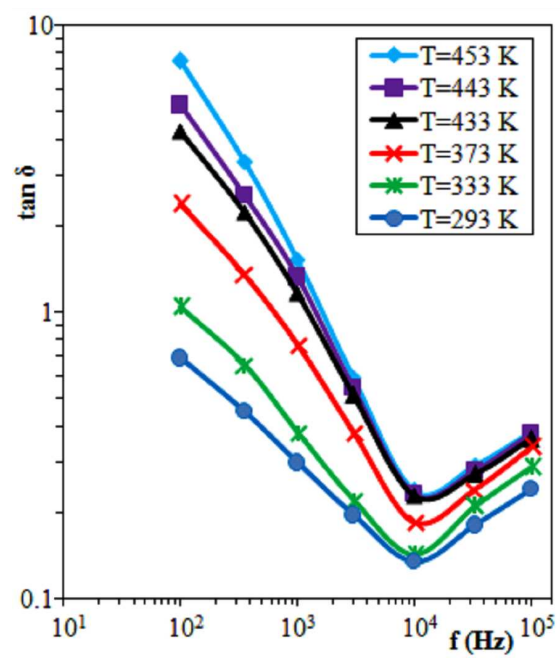

Fig. 11. Variation of loss factor with frequency at different temperatures.

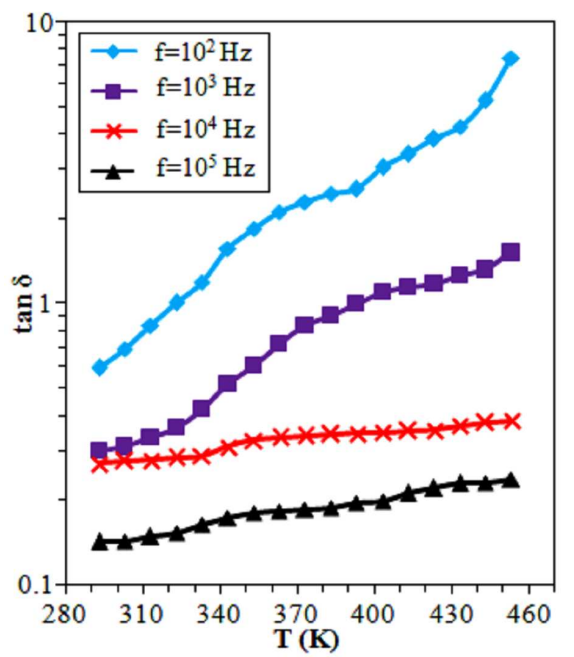

Fig. 12. Variation of loss factor with temperature at different frequencies.

Equation (6) predicts that at high frequency region the $\omega$ term is dominant whereas at low frequency region the term containing $1 / \omega$ is dominant. The angular frequency 
at which loss factor reaches the least value is calculated to be [28]:

$$
\omega_{\min }=\left(\frac{1}{r R C^{2}}\right)^{1 / 2} \text {. }
$$

It is seen in Fig. 12 that there is a gentle increase in loss factor as the temperature increases for $f \geq 10^{4} \mathrm{~Hz}$, however a quite sharp increase in loss factor is observed for $f<10^{4} \mathrm{~Hz}$.

\section{Conclusions}

$\mathrm{AC}$ measurements of sandwich devices in the form of $\mathrm{Al} / \mathrm{CuPc} / \mathrm{Au}$ were carried out in temperature range of $293-453 \mathrm{~K}$ and frequency range of $10^{2}-10^{5} \mathrm{~Hz}$. FESEM images and XRD micrographs were studied to investigate the morphology of the samples. The XRD micrograph indicates the configuration of $\alpha-\mathrm{CuPc}$ with the (510) plane as the preferred orientation. Optical band-gap energy of $\mathrm{CuPc}$ thin film was determined to be $2.81 \pm 0.01 \mathrm{eV}$. At relatively high temperatures $(T>413 \mathrm{~K})$ and low frequencies $\left(f<10^{4} \mathrm{~Hz}\right)$, capacitance seems to be strongly dependent on frequency, but at lower temperature and higher frequency range it is generally independent of frequency. Loss factor decreased considerably with increasing frequency to a minimum value at about $f=10^{4} \mathrm{~Hz}$ and increased afterwards. The activation energy decreases with increasing frequency and has a larger value at higher temperatures. The conductivity increases quite noticeably with increasing frequency particularly for $T \leq 413 \mathrm{~K}$. Exposure to oxygen was found to have a profound effect on the conductivity of samples. It is evident that in the low frequency range, conductivity is strongly dependent on oxygen exposure time. Although annealing decreased the conductivity of the samples significantly, full reversibility was not obtained. It is probable that prolonged annealing and/or higher annealing temperature can totally reverse the effects of oxygen adsorption. The AC electrical characteristics are in good agreement with Goswami and Goswami model. At low temperatures conduction mechanism is dominated by hopping-type process, whereas at high temperatures conduction is more likely to be via a band-type transport process.

\section{References}

[1] C.J. Liu, J.J. Shih, Y.H. Ju, Sens. Actuators B 99, 344 (2004).

[2] T. Basova, A. Tsargorodskaya, A. Nabok, A.K. Hassan, A.G. Gurek, G. Gumus, V. Ahsen, Mater. Sci. Eng. C 29, 814 (2009).

[3] A.K. Abass, R.A. Collins, A. Krier, J. Phys. Chem. Solids 54, 375 (1993).
[4] W. Chao, X. Zhang, C. Xiao, D. Liang, Y. Wang, J. Colloid Interface Sci. 325, 198 (2008).

[5] J. Wang, W.J. Blau, Appl. Phys. B 91, 521 (2008).

[6] E.M. El-Menyawy, H.M. Zeyada, M.M. El-Nahass, Solid State Sci. 12, 2182 (2010).

[7] F. Yakuphanoglu, M. Arslan, Solid State Commun. 132, 229 (2004).

[8] A. Krier, M.E. Azim-Araghi, J. Phys. Chem. Solids 58, 711 (1997).

[9] M.E. Azim-Araghi, M.J. Jafari, Eur. Phys. J. Appl. Phys. 52, 10402 (2010).

[10] M.E. Azim-Araghi, E. Karimi-Kerdabadi, M.J. Jafari, Eur. Phys. J. Appl. Phys. 55, 30203 (2011).

[11] M.M. El-Nahass, F.S. Bahabri, A.A. Al-Ghamdi, S.R. Al-Harbi, Egypt. J. Sol. 25, 307321 (2002).

[12] H.S. Lee, M.W. Cheon, Y.P. Park, Trans. Electr. Electron. Mater. 12, 40 (2011).

[13] M.I. Newton, T.K.H. Starke, G. McHale, M.R. Willis, Thin Solid Films 360, 10 (2000).

[14] Y.L. Lee, C.Y. Hsiao, R.H. Hsiao, Thin Solid Films 468, 280 (2004).

[15] A. Oprea, U. Weimar, E. Simon, M. Fleischer, H.P. Frerichs, Ch. Wilbertz, M. Lehmann, Sens. Actuators B 118, 249 (2006).

[16] A.K. Hassan, R.D. Gould, J. Phys., Condens. Matter 1, 6679 (1989).

[17] M.T. Robinson, G.E. Klein, J. Am. Chem. Soc. 74, 6294 (1952).

[18] B.B. He, Two-dimensional X-ray Diffraction, Wiley Hoboken, NJ 2009, p. 191.

[19] A.K. Hassan, R.D. Gould, Phys. Status Solidi A 132, 91 (1992).

[20] M.J. Stillman, T.N. Nyokong, in: Phthalocyanines: Properties and Applications, Vol. 1, Eds. C.C. Lenznoff, A.B.P. Lever, VCH, New York 1989, Ch 3, p. 133.

[21] S.B. Brown, Introduction to Spectroscopy for Biochemistry, Academic Press, London 1980.

[22] S. Mathew, C. Sudarsanakumar, C.S. Menon, Optoelectron. Adv. Mater.-Rapid Commun. 1, 614 (2007).

[23] M.E. Azim-Araghi, D. Campbell, A. Krier, R.A. Collins, Semicond. Sci. Technol. 11, 39 (1996).

[24] M.E. Azim-Araghi, Indian J. Pure Appl. Phys. 45, 40 (2007).

[25] P.J Harrop, D.S. Campbell, in: Handbook of Thin Film Technology, Eds. L.I. Maissel, R. Glang, McGraw Hill, New York 1983, Ch. 16, p. 16.

[26] H.R. Kerp, K.T. Westerdui, A.T. van Veen, E.E. van Faasseu, J. Mater. Res. 16, 503 (2001).

[27] J.D. Wright, Prog. Surf. Sci. 31, 1 (1989).

[28] A. Goswami, A.P. Goswami, Thin Solid Films 16, 175 (1973). 